\title{
НЕКОТОРЫЕ ОСОБЕННОСТИ СВОЙСТВ НАНОСТРУКТУРИРОВАННЫХ МАТЕРИАЛОВ КАК УЧАСТНИКОВ ХИМИЧЕСКИХ ПРОЦЕССОВ
}

\author{
(C) 2018 В. И. Вигдорович ${ }^{1,2}$ \\ ${ }^{1}$ Всероссийский научно-исследовательский институт использования техники и нефтепродуктов в сельском \\ хозяйстве, Ново-Рубежный пер., 28, 392022 Тамбов, Россия \\ ${ }^{2}$ Тамбовский государственный технический университет, ул. Советская, 106, 392000 Тамбов, Россия \\ e-mail:vits21@mail.ru
}

Поступила в редакцию 02.02.2018

\begin{abstract}
Аннотация. Рассмотрен ряд особенностей термодинамических закономерностей, свойственных наноструктурированным материалам, и характер зависимости энергии активации процессов с их участием от эффективного размера частиц нановещества. В этих условиях эффективный размер частиц ( $a$ ) или число элементарных составляющих малоатомных кластеров (атомы, молекулы, радикалы) становятся термодинамическим параметром. В частности, химический потенциал вещества в случае наноразмерных частиц является функцией их эффективного размера или более строго - функцией концентраций вакансий $C_{\mathrm{a}}$. Величина $C_{\mathrm{a}}$, в свою очередь, определяется значением $a$, уровнем поверхностной энергии и изменением объема частиц, возникающим в результате замены в них атома вакансией. Энергия активации процессов $\left(E_{\mathrm{A}}\right)$, в которых участвуют наночастицы (прямые и обратные химические реакции, явления адсорбции и десорбции как их разновидности), становится функцией величины $a$ наноструктурированных частиц. Подобное явление свойственно для самого различного характера энергетической активности адсорбционных центров. Оно приводит к возрастанию роли флуктуаций и случайных процессов и к появлению точки бифуркации на зависимости $E_{\mathrm{A}}=f(a)$, начиная с которой возможны различные направления процессов, ведущих к кажущемуся разбросу экспериментальных данных. Наблюдаемая картина, в определенной мере, - некий аналог принципа неопределенности Гейзенберга с наличием и существенных отличий от него.
\end{abstract}

Ключевые слова: наноструктура, термодинамика, энергия активации, бифуркация, направление процесса.

DOI: https://doi.org/10.17308/kcmf.2018.20/512

\section{ВВЕДЕНИЕ}

Ранее нами неоднократно указывалось на многочисленные особенности физико-химических свойств и поведения наноструктурированных материалов. Характеризующие их особенности, как правило, играют решающую роль в процессах, протекающих в наносистемах, и определяют функциональные свойства объектов индустрии наносистем [1-5].

Прежде всего, укажем на наличие некоторого наносостояния вещества [1], характерного для определённых идеализированных условий существование наносистемы, когда поверхность наночастиц не взаимодействует с окружающей средой. В первом приближении, это некоторое промежуточное состояние вещества между существованием его в виде отдельных атомов и молекул с их очень малой энергией кооперативного взаимодействия и, напротив, в виде конденсированной фазы с размером частиц более 100 нм. Это состояние, когда многократно повышена реакционная способность активных центров, но которое крайне неустойчиво. Подобными являются системы типа, например, метастабильной свежеобразованной поверхности металла, ювенильные высокоактивные центры которой быстро блокируются молекулами веществ, адсорбирующимися из окружающей среды [2-4].

Несомненно, что всякое взаимодействие наноструктурированых веществ (НСВ) независимо от их химической природы имеет общие термодинамические особенности. Дело в том, что применительно к НСВ эффективный размер частиц ста- 


\section{В. И. ВИГДОРОВИЧ}

новиться термодинамическим параметром, от которого зависят свойства вещества также или даже более существенно, чем, например, от температуры. В частности, химический потенциал вещества $\left(\mu_{\infty}\right)$ при некотором избранном стандартном состоянии, выбираемом достаточно произвольно, является постоянным только для массивных частиц с эффективным параметром $a>100$ нм. В наноразмерной области дисперсности вещества при прочих неизменных условиях действительна зависимость [3-5]:

$$
\mu_{i}=\mu_{i, \infty}+f_{i}(a), i=\overline{1 \ldots k},
$$

где $a$ - термодинамический параметр.

Принципиальное решение проблемы наноразмерности предложено в [6]. Постулировано, что поверхностная энергия является функцией дисперсности частиц и наличия дефектов. А связь концентрации вакансий с размером частиц передается выражением:

$$
C_{a}=C_{\infty} \exp \left[(2 \sigma / a)\left(\Delta V / k_{\mathrm{b}} T\right)\right],
$$

где $C_{a}$ и $C_{\infty}$ - соответствующие средние концентрации вакансий в частицах с эффективным параметром $a$ и в массивном образце, $\sigma-$ поверхностное натяжение, $\Delta V$ - изменение объема при замене атома вакансией, $k_{\text {ь }}$ - константа Больцмана. Остальные обозначения общепринятые. Величина $a$ играет роль термодинамического параметра [6]. При $a \leq 10$ нм вклад концентрации вакансий становится существенным.

При диспергировании появляется дополнение $\Delta \Delta G$, равное [6]:

$$
\delta G=\frac{2 M \delta}{\rho} N_{\mathrm{A}} d-k_{\mathrm{B}} T\left(C_{\mathrm{A}}-C_{\infty}\right),
$$

т. е. $\delta G=\Delta \Delta G$, где $M$ - молярная масса, $\rho-$ плотность, $C_{i}$ - удельная концентрация вакансии (в расчете на атом), $N_{\text {А }}$ - число Авогадро.

Тогда по [6], величина изменения свободной энергии Гиббса $\Delta G_{p}$ для реакции типа $v_{\text {и }} A \rightarrow v_{\Pi} B$ передается зависимостью:

$$
\Delta G_{i}=\Delta G_{a}+\sum_{i=1}^{n} \mathrm{v}_{n, i} \delta G_{n, i}-\sum_{i=1}^{n} \mathrm{v}_{u, i} \delta G_{u, i}
$$

и $\Delta G_{\text {массива }}>\Delta G_{a}$, следовательно, возможно изменение знака $\Delta G$ и направление самопроизвольного течения процесса, $v_{n}$ и $v_{u}$ - стехиометрические коэффициенты соответственно продуктов реакции и исходных веществ [6]. По оценкам [6] $\Delta \Delta G$ находится в пределах 10...100 кДж/моль.

Согласно [6], в случае эффективных малых размеров частиц функции $f(a)$ можно придать вид:

$$
f(\mathrm{a})=\left\{k_{k}\left[C_{\infty} \exp (2 \sigma / \mathrm{a})\left(\Delta V / k_{\mathrm{b}} T\right)\right]\right\},
$$

где $k_{k}-$ коэффициент корреляции, принимающий значения -1 или +1 в зависимости от параметра изменения термодинамического характера.

Тогда широко известное уравнение первого начала термодинамики, когда помимо механической работы имеет место превращения вещества [7]:

$$
\delta Q=d u+p d v+\sum_{i=1}^{k} \mu_{i} d n_{i}
$$

принимает вид

$$
\delta Q=d u+p d v+\sum_{i=1}^{k}\left[\mu_{i, \infty}+f_{i}(a)\right] d n_{i} .
$$

Для того же случая вместо уравнения [7]:

$$
d U=T d S-p d v+\sum_{i=1}^{k} \mu_{i} d n_{i} .
$$

Следует использовать зависимость [3-5]:

$$
\delta U=T d S-p d v+\sum_{i=1}^{k}\left[\mu_{i, \infty}+f_{i}(a)\right] d n_{i}
$$

Следовательно, изменение внутренней энергии системы связано с эффективным размером частиц любого из претерпевающих превращение веществ. При этом в качестве химического взаимодействия следует рассматривать не только процессы типа:

$$
a A+b B \rightarrow c C+d D,
$$

но и адсорбционные явления, взаимную растворимость веществ в твёрдом состоянии и многие смежные с ними.

Те же особенности характерны для связи изменения свободной энергии Гельмгольца $(F)$ и Гиббса $(G)$ с термодинамическими параметрами системы:

$$
\begin{gathered}
d F=-p d v-S d T+\sum_{i=1}^{k} \mu_{i} d n_{i}, \\
d G=V d p-S d T+\sum_{i=1}^{k} \mu_{i} d n_{i}
\end{gathered}
$$

и др., когда в случае наноструктурированных систем последний член правой части уравнения должен быть учтён в виде:

$$
\sum_{i=1}^{k}\left[\mu_{i, \infty}+f_{i}(a)\right] d n_{i}
$$

Известно, что малоатомные кластеры играют подчас решающую роль в процессах с участием наноразмерных частиц [8]. Согласно [9], уравнение (1) для подобных кластеров $\mathrm{Cu}_{\rho}, \mathrm{Ag}_{\rho}, \mathrm{Au}_{\rho}$ принимает вид:

$$
\mu_{i}=\mu_{i, \infty}+f_{i}(\rho)
$$

где $\rho$ - число атомов в малоатомном кластере. 
Существенное влияние должно, несомненно, оказывать наносостояние вещества и на кинетику процессов, протекающих с его участием. Можно рассмотреть влияние эффективных размеров наночастиц на кинетические показатели процессов константы скоростей прямых или обратных реакций, коэффициенты диффузии, состав промежуточных комплексов и т.д. Но целесообразнее оценивать влияние эффективных параметров наноразмерных частиц на интегральный кинетический фактор энергию активации $E_{\mathrm{A}}$. Известно, что величина $E_{\mathrm{A}}$ подчиняется уравнению Аррениуса [10].

$$
E_{\mathrm{A}}=-\ln (k / B) R T
$$

где $k$ - константа скорости, $T$ - абсолютная температура, $B$ - предэкспоненциальный множитель, $R$ - универсальная газовая постоянная. Отметим, что $B$ и $E_{\mathrm{A}}$ не зависят или почти не зависят от температуры в определённом её интервале. Однако подобная картина имеет место лишь для макроразмерных ( $a>100$ нм) участников химических процессов.

В случае наноструктурированых систем при $T=$ const несомненно, наличие зависимости:

$$
E_{\mathrm{A}}=f(a) \text {. }
$$

Возможные варианты зависимости (3) графически представлены на рис. 1. Их вид вытекает из данных рис. 2 и более широко из результатов, приведенных в [10].

Рассмотрим вид зависимости (3) более подробно. На рис. $1 k$ (сплошная линия) и рис. $1 m$ имеет место снижение величины $E_{\mathrm{A}}$ с уменьшением эффективного размера частиц. Одновременно на рис. $1 k$ (пунктир) и $1 n$ наблюдается повышение $E_{\text {А }}$ с уменьшением эффективного размера $a$. Рис. $1 k$ характеризует скачкообразный, рис. $1 m$ и $1 n-$ протяженный тип зависимости $E_{\mathrm{A}}$ от $a$.
На рис. $1 k$ в области величин $a$, соответствующих участку $A B$, взаимодействуют микро- или наноструктуры $(a<100$ нм), которые не обладают наноэффектом. При этом под наноэффектом понимается влияние эффективного параметра частиц на $E_{A}$, обусловленную энергетикой процесса, а не их абсолютными значениями эффективных размеров или удельной поверхности $\left(S_{\text {ул }}\right)$, которые связаны между собой зависимостью $[11,12]$ :

$$
S_{\text {уд }}=\gamma / a \text {, }
$$

где $S_{\text {уд }}$ характеризует отношение эффективной поверхности частиц к их объему, $\gamma$ - безразмерная константа, определяемая формой частиц, $a-$ их эффективный параметр, как определено ранее. В точках, соответствующих $a=L$ или $a=M$ (рис. $1 k$ ), происходит резкое изменение рассматриваемого кинетического параметра, в данном случае $E_{\text {A }}$. При этом $L$ и $M$ не являются фундаментальными константами, независимыми от природы систем и условий, при которых протекает взаимодействие.

С последующими изменениями $a$, т. е. при $a<L$ или $a<M$ (рис. $1 k$ ) система наноструктурирована и находится в наносостоянии, которое нарушается внешним воздействием (в частности, адсорбцией микропримесей воздуха). Завершается это состояние по мере снижения величины $a$ переходом к атомным и молекулярным системам (газы).

Легко видеть, что рассматриваемый характер $E$, может, в принципе, привести как к возрастанию, так и к снижению энергии активации процесса. В технологическом плане интересны оба маршрута. Маршрут $A B C D$ (рис. $1 k$ ) ведёт к повышению скорости реакции и, следовательно, интересен по отношению к процессам, связанным с получением целевого продукта (ЦП). Маршрут $A^{\prime} B^{\prime} C^{\prime} D^{\prime}$ (рис. $1 k$ ) является позитивным по отношению к скорости накопления побочных продуктов, (ПП), снижающих
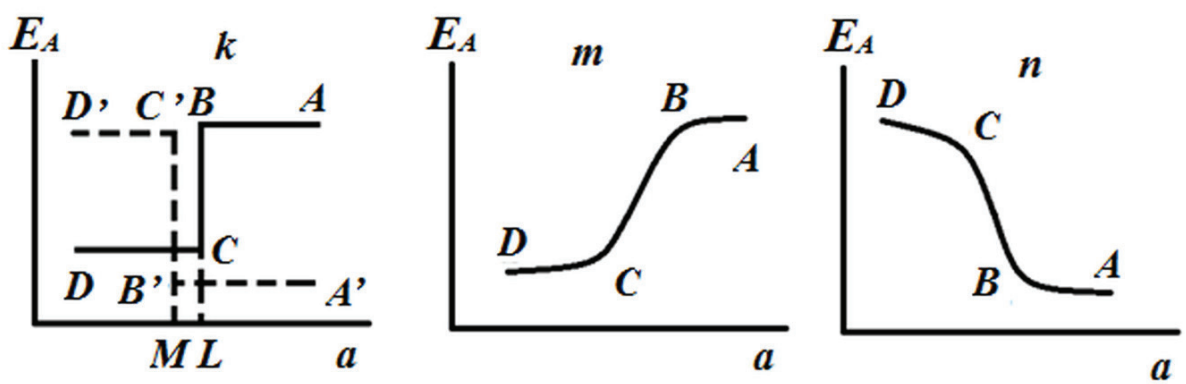

Рис. 1. Возможный характер зависимости энергии активации процесса от эффективного размера наноразмерных частиц при $T=$ const

[Fig. 1. A possible character of the dependence of the activation energy of the process on the effective size of nanoscale particles at $T=$ const] 


\section{В. И. ВИГДОРОВИЧ}

выход по ЦП и способных к образованию высокотоксичных ПП.

Вместе с тем, вариант, показанный на рис. $1 k$, является лишь частным случаем. Более вероятны зависимости, приведённые на рис. $1 m$ и $1 n$. Характерный, экспериментально наблюдаемый пример подобной зависимости приведен на рис. 2 [10].

Следует отметить, что в силу неустойчивости наносостояния системы процессы с участием наноструктурированых материалов протекают вдали от равновесного (стационарного) состояния. Подобная ситуация приводит к значительному возрастанию роли флуктуаций и случайных процессов, в частности, связанных с адсорбцией продуктов из атмосферного воздуха, носящих стохастический характер. Такая картина достаточно подробно проанализирована в [7] на примере сравнительно простого случая бифуркаций, как общего свойства процессов, описываемых нелинейными дифференциальными уравнениями, имеющими несколько случайных решений.

Согласно [7], при нахождении системы в состоянии, далеком от равновесия, устойчивость направления процесса не обеспечивается. Необходимые условия неустойчивости системы рассмотрены в [7]. Вдали от области, близкой к равновесию, что характерно для наноразмерных частиц, принимающих участие в химических процессах, возможно наличие множества состояний и отсутствие про-

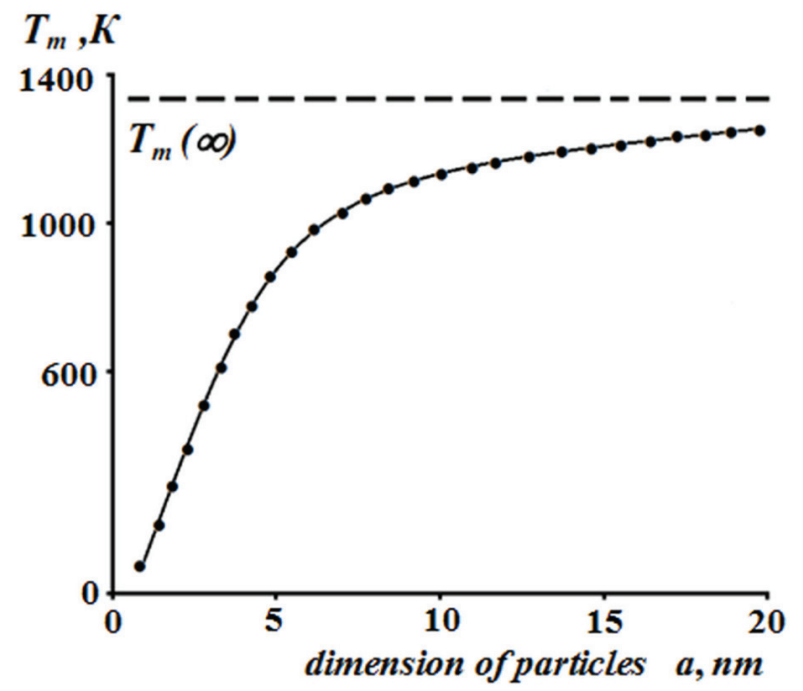

Рис. 2. Зависимость температуры плавления золота от размера частиц. Точки экспериментальные данные, сплошная линия - расчёт [12]

[Fig. 2. Dependence of the melting point of gold on the particle size. Points are experimental data, solid line calculation [12]] гнозируемости. Согласно [7], потерю устойчивости неравновесного состояния можно проанализировать, исходя из устойчивости нелинейных дифференциальных уравнений. При этом используются бифуркации, как в данном случае ветвление решений дифференциальных уравнений, характеризующих различное направление протекания процесса. Это можно проиллюстрировать на нелинейном дифференциальном уравнении [7]:

$$
\frac{d \alpha}{d v}=-\alpha^{3}+\lambda \alpha
$$

где $\lambda$ - некий параметр, стационарные решения имеют вид:

$$
\begin{aligned}
& \text { 1. } \alpha=0 \\
& \text { 2. } \alpha= \pm \sqrt{\frac{\lambda}{3}}
\end{aligned}
$$

Причем второе решение не одновариантно, что очень важно для неравновесных систем с участием наноразмерных частиц. Согласно [7], если при $\lambda<$ 0 имеется одно решение уравнения (4), при $\lambda>0-$ их уже три (рис. 3)

При $\lambda>0$ решение разветвляется, следовательно, точка с $\lambda=0$ является точкой бифуркации. По [7], стационарное решение $\alpha_{S}$ устойчиво, если возмущения $\delta(\tau)$ мало ( $\tau-$ время от начала действия $\delta)$ и быстро затухает во времени.

Согласно многим экспериментальным данным эта картина носит вообще постоянный либо часто наблюдаемый характер для наносистем, в силу чего имеет место достаточно широкий разброс экспериментальных данных. Так, по [13-18], емкость углеродных нанотрубок при абсорбции водорода меняется от 0.8 до 12 масс.\%. Минимальная емкость многостенных УНТ (МУНТ) наблюдается в [13], максимальная - в [18].

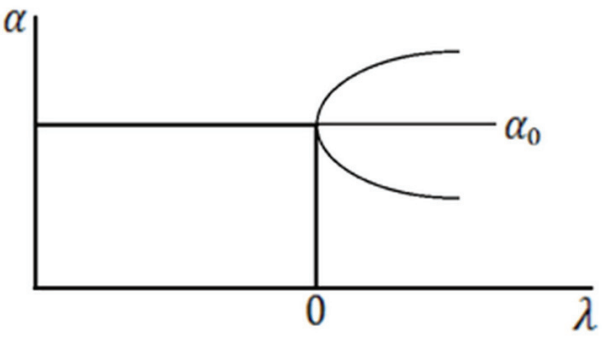

Рис. 3. Ветвление решений уравнений при $\alpha=0$ (без ветвления) и $\alpha= \pm \sqrt{\lambda}$ как функции величины $\lambda[6]$

[Fig. 3. The branching of the solutions of the equations at $\alpha=0$ (without branching) and $\alpha= \pm \sqrt{\lambda}$ as a function of $\lambda[6]]$ 
Подобная картина часто приводит исследователей, в целом, к мнению, что в большинстве случаев емкость МУНТ мала, и углеродные нанотрубки неперспективны как аккумуляторы водорода. Однако это, видимо, не так, и наблюдаемые закономерности нужно интерпретировать не как разброс экспериментальных данных, а как результат подобных бифуркаций (рис. 3) с последующим учётом и использованием этого явления в технологических целях. Иначе можно излишне негативно отнестись ко многим нанопродуктам при планировании создания в будущем индустрии наносистем.

Необходимо отметить, что возможное наличие бифуркаций не должно явиться причиной отказа от использования наноструктурированых систем в рамках наноиндустрии.

Вместе с тем, бифуркации, видимо, являются элементом квантования (рис. 3).

\section{ЗАКЛЮЧЕНИЕ}

Для проявления наноструктурироваными материалами специфического термодинамического или кинетического эффектов необходимо достижение и поддержание ими наносостояния вещества.

В связи с тем, что процессы с участием наноструктурированых материалов протекают вдали от равновесия, для них характерны стохастически реализуемые различные маршруты наработки конечных целевых продуктов. При изучении и использовании процессов с участием наноструктурированых продуктов необходимо учитывать возможность бифуркационных явлений.

Исследование выполнено за счет гранта Российского научного фонда (проект № 18-1600006).

\section{СПИСОК ЛИТЕРАТУРЫ}

1. Вигдорович В. И., Цыганкова Л. Е., Осетров А. Ю. // Физикохимия поверхностей и защита материалов, 2011, т. 47, № 3, с. 330-336. DOI: $10.1134 /$ S207020511103018X

2. Вигдорович В. И., Стрельникова К. О. // Физикохимия поверхностей и защита материалов, 2012, т. 48, № 3, с. 316-320. DOI: 10.1134/S2070205112030197
3. Вигдорович В. И., Цыганкова Л. Е. Физикохимия наноструктурированых наноматериалов. 2012, Тамбов, Изд-во Першина Р. В., 234 с.

4. Вигдорович В. И., Цыганкова Л. Е., Шель Н. В, Бернацкий П. Н. Теоретические и прикладные вопросы нанотехнологий (Современное состояние и проблемы). 2016, Тамбов, Изд-во Першина Р. В, 186 с.

5. Вигдорович В. И., Цыганкова Л. Е. // Физикохимия поверхностей и защита материалов. 2012, т. 48, № 5, c. 415-421. DOI: $10.1134 / \mathrm{S} 2070205112050152$

6. Лидоренко Н. С., Чижик С. П., Гладких Н. П., Григорьева Л. К., Куклин Р. Н. // Докл. АН СССР, 1981, т. 257, № 5, с. 1114-1116.

7. Пригожин И., Кондепуди Д. Современная термодинамика. От тепловых двигателей до диссипативных структур. М.: Мир, 2009, 461 с.

8. Гамбург Ю. Д. Электрохимическая кристаллизация металлов и сплавов. М.: Янус-К, 1997, 384 с.

9. Вигдорович В. И., Цыганкова Л. Е., Шель Н. В. // Физикохимия поверхностей и защита материалов, 2015, т. 51, № 4, с. 420-427. DOI: 10.1134/S207020511 5040346

10. Штиллер В. Уравнение Аррениуса и неравновесная кинетика. М.: Мир, 2000, 175 с.

11. Воюцкий С. С. Курс коллоидной химии. М.: Химия, 1964,575 с.

12. Сергеев Г. Б. Нанохимия. М.: Изд-во МГУ, 2007, $336 \mathrm{c}$.

13. Nutzenadel C., Zuttel A., Chartouni D., Schlapbach L. // Electrochem. Solid-State Lett., 1999, vol. 2, № 1, pp. 30-32. DOI: 10.1149/1.1390724

14. Qin X., Gao X. P., Liu H., Yuan D. Y., Gong W. L. // Electrochem. Solid-State Lett., 2000, vol. 2, № 3, pp. 532535. DOI: $10.1149 / 1.1391200$

15. Rajalakshmi N., Dhathathreyan K. S., Govindaraj A., Satishkumar B. C. // Electrochem. Acta, 2000, vol. 45, pp. 4511-4515. DOI: https://doi.org/10.1016/S00134686(00)00510-7

16. Fazle Kibria A. K. M. Mo Y. H., Park K. S., Nahm K. S., Yun M. Y. // Int. J. Hydrogen Energy, 2001, vol. 26, pp. 823-829. DOI: https://doi.org/10.1016/S03603199(01)00007-6

17. Gundiah G., Govindaraj A., Rajalakshmi N., Dhathathreyan K. S., Rao C. N. R. // J. Mater. Chem., 2003, vol. 13, pp. 209-213. DOI: 10.1039/b207107j

18. Солодкова Л. Н., Ляхов Б. Ф. Липсон А. Г., Цивадзе А. Ю. // Физикохимия поверхностей и защита материалов, 2010, т. 46, №. 5, с. 450-453. DOI: 10.1134/ S2070205110050035 


\title{
SOME PROPERTY FEATURES OF NANOSTRUCTURED MATERIALS AS PARTICIPANTS OF CHEMICAL PROCESSES
}

\author{
(C) 2018 V. I. Vigdorovich ${ }^{1,2}$ \\ ${ }^{1}$ All-Russian Scientific Research Institute for the Use of Machinery and Oil Products in Agriculture, \\ 28 Novo-Rubezhny per., 392022 Tambov, Russia \\ ${ }^{2}$ Tambov State Technical University, 106 Sovetskaya str., Tambov, Russia \\ e-mail: vits21@mail.ru
}

Received 02.02.2018

\begin{abstract}
The article considers a number of features of thermodynamic regularities peculiar to nanostructured materials and the nature of the dependence of the activation energy of processes involving nanostructured materials on the effective particle size of the nanosubstance. Under these conditions, the effective particle size $(a)$ or the number of elementary components of low-atom clusters (atoms, molecules, and radicals) becomes a thermodynamic parameter. In particular, the chemical potential of a substance in case of nanoscale particles is a function of their effective size, or more strictly, a function of the concentrations of vacancies in $C_{\mathrm{a}}$. The value of $C_{\mathrm{a}}$, in its turn, is determined by the value of $a$, the level of the surface energy, and the change in the volume of particles that results from the replacement of the atom by a vacancy in them. The activation energy of processes $\left(E_{\mathrm{A}}\right)$ in which nanoparticles participate (direct and reverse chemical reactions, adsorption and desorption phenomena as their varieties) become a function of the magnitude of nanostructured particles. A similar phenomenon is characteristic of adsorption centres' energy activity of diverse nature. It leads to an increase in the role of fluctuations and random processes and to the appearance of a bifurcation point on the dependence $E_{\mathrm{A}}=f(a)$, from which various directions of the processes, leading to an apparent scatter of the experimental data, are possible. The observed picture, to a certain extent, is similar to the Heisenberg uncertainty principle. At the same time it has some significant differences.
\end{abstract}

Keywords: nanostructure, thermodynamics, activation energy, bifurcation, direction of the process.

DOI: https://doi.org/10.17308/kcmf.2018.20/512

\section{ACKNOWLEDGEMENTS}

The research was carried out at the expense of a grant from the Russian Science Foundation (project No. 18-16-00006).

\section{REFERENCES}

1. Vigdorovich V. I., Tsygankova L. E, Osetrov A. Yu. Protection of Metals and Physical Chemistry of Surfaces, 2011, vol. 47, no. 3, pp. 410-415. DOI: 10.1134/ S207020511103018X

2. Vigdorovich V. I., Strelnikova K. O. Protection of Metals and Physical Chemistry of Surfaces, 2012, vol. 48, no. 3, pp. 383-387. DOI: 10.1134/S2070205112030197

3. Vigdorovich V. I., Tsygankova L. E. Fizikokhimiya nanostrukturirovanykh nanomaterialov [Physicochemistry of Nanostructured Nanomaterials]. Tambov, 2012, Publishing house Pershina R. V., 234 p. (in Russ.)

4. Vigdorovich V. I., Tsygankova L. E, Shel N. V, Bernatsky P. N. Teoreticheskie i prikladnye voprosy nanotekhnologii (Sovremennoe sostoyanie $i$ problem)
[Theoretical and Applied Problems of Nanotechnology (Current State and Problems)]. Tambov, Publishing house Pershina R. V., 2016, 186 p. (in Russ.)

5. Vigdorovich V. I., Tsygankova L. E. Protection of Metals and Physical Chemistry of Surfaces, 2012, vol. 48, no. 5, pp. 501-507. DOI: 10.1134/S2070205112050152

6. Lidorenko N. S., Chizhik S. P., Gladkikh N. P., Grigorieva L. K., Kuklin R. N. Dokl. An USSR, 1981, vol. 257, no. 5, pp. 1114-1116. (in Russ.)

7. Kondepudi D., Prigogine I. Modern Thermodynamics: From Heat Engines to Dissipative. John Wiley \& Sons, 1998, $486 \mathrm{p}$.

8. Hamburg Yu. D. Elektrokhimicheskaya kristallizatsiya metallov i splavov [Electrochemical Crystallization of Metals and Alloys]. Moscow, Janus-K Publ., 1997, 384 p.

9. Vigdorovich V. I., Tsygankova L. E., Shel N. V. Protection of Metals and Physical Chemistry of Surfaces, 2015, vol. 51, no. 4, pp. 567-574. DOI: 10.1134/S2070205115040346

10. Stiyler B. Uravnenie Arreniusa i neravnovesnaya kinetika [The Arrhenius equation and the Unequal Kinetics]. Moscow, Mir Publ., 2000, 175 p. (in Russ.) 
11. Voyutskiy S. S. Kurs kolloidnoi khimii [Course of Colloid Chemistry]. Moscow, Khimiya Publ., 1964, 575 p. (in Russ.)

12. Sergeev G. B. Nanokhimiya [Nanochemistry]. Moscow, Publishing house of the Moscow State University, 2007, 336 p. (in Russ.)

13. Nutzenadel C., Zuttel A., Chartouni D., Schlapbach L. Electrochem. Solid-State Lett., 1999, vol. 2, no. 1, pp. 30-32. DOI: 10.1149/1.1390724

14. Qin X., Gao X. P., Liu H., Yuan D. Y., Gong W. L. Electrochem. Solid-State Lett., 2000. vol. 2, no. 3, pp. 532535. DOI: 10.1149/1.1391200

15 Rajalakshmi N., Dhathathreyan K. S., Govindaraj A. Satishkumar B. C. Electrochem. Acta, 2000, vol. 45, pp. 4511-4515. DOI: https://doi.org/10.1016/S00134686(00)00510-7

16 Fazle Kibria A. K. M. Mo Y. H., Park K. S., Nahm K. S., Yun M. Y. Int. J. Hydrogen Energy, 2001, vol. 26, pp. 823-829. DOI: https://doi.org/10.1016/S03603199(01)00007-6

17 Gundiah G., Govindaraj A., Rajalakshmi N., Dhathathreyan K. S., Rao C. N. R. J. Mater. Chem., 2003, vol. 13, pp. 209-213. DOI: 10.1039/b207107j

18. Solodkova L. N, Lyakhov B. F., Lipson A. G, Tsivadze A. Yu. Protection of Metals and Physical Chemistry of Surfaces, 2010, vol. 46, no. 5, pp. 524-527. DOI: 10.1134/ S2070205110050035
Вигдорович Владимир Ильич - д. х. н., професcop, академик РАЕН, заслуженный деятель науки и техники РФ, главный научный сотрудник ВНИИТиН, профессор Тамбовского государственного технического университета по совместительству; тел./факс: +7(4752) 446414, e-mail: vits21@mail.ru
Vladimir I. Vigdorovich - Dr. Sci. (Chem.), Professor, Academician of the Russian Academy of Natural Sciences, Honored Worker of Science and Technology of the Russian Federation, Chief Scientific Officer of VNIITiN, Professor of Tambov State Technical University in combination; tel./fax: +7(4752) 446414, e-mail: vits21@mail.ru 